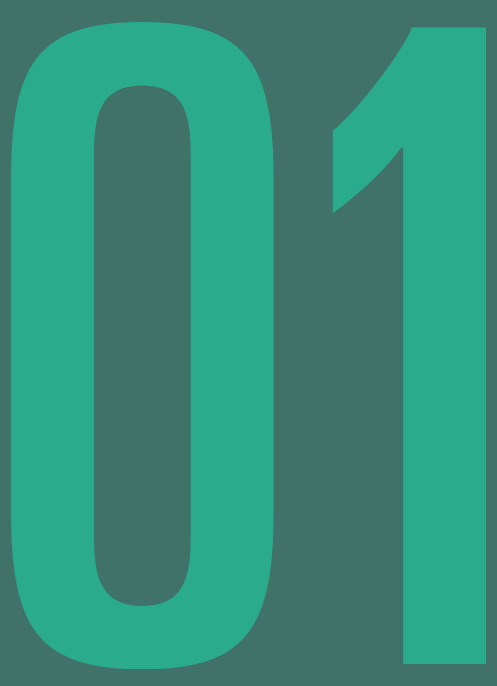

\title{
VALIDACIÓN DE INSTRUMENTO PARA MEDIR EL APROVECHAMIENTO DE LOS RECURSOS ASOCIADOS A LAS TIC EN INSTITUCIONES DE EDUCACIÓN SUPERIOR
}

\section{Validation of an instrument to measure the use of resources associated with ICT in institutions of higher education}

MCI. Miguel Angel Fitch Osuna'

miguelfosuna@gmail.com cDr. Filosofía con especialidad en Administración. Universidad Autónoma de Nuevo León Maestro en Ciencias de la Ingeniería. Universidad Autónoma de Nuevo León

Dra. María de Jesús Araiza Vázquez ${ }^{2}$ Instructional Technology and Distance Education. Nova Southeastern University 
Palabras clave: Aprovechamiento de las TIC, Competencias digitales, Autoeficacia. Keywords: Use of ICT, Digital skills, Self-efficacy.

\section{Resumen:}

El propósito de este estudio consiste en validar una serie de indicadores que nos permiten evaluar el aprovechamiento de los recursos tecnológicos en instituciones de nivel superior, en este caso enfocados a la licenciatura de Arquitectura y Diseño Industrial. La muestra corresponde a 173 estudiantes que se encontraban próximos a presentar su examen de egreso. Para validar el modelo se han utilizado sistema de ecuaciones estructurales, teniendo 7 constructos, los cuales nos referiremos como: Acceso de recursos TIC, Competencias digitales del estudiante, competencias generales del docente, competencias digitales del estudiante, actitudes de estudiante ante las TIC, autoeficacia percibida y aprovechamiento de los recursos TIC. Las universidades interesadas pueden utilizar este instrumento para diagnosticar y mejorar las características tanto de su recurso humano como de infraestructura tecnológica. Los resultados obtenidos muestran la confianza de ser un instrumento válido y fiable para la medición de las variables de estudio.

\section{Abstract}

The purpose of this study is to validate a series of indicators that allow us to evaluate the use of technological resources in higher level institutions, in this case focused on the degree of Architecture and Industrial Design. The sample corresponds to 173 students who were about to present their term exam. To validate the model, a system of structural equations has been used, having 7 constructs, which we will refer to as: Access to ICT resources, digital competences of the student, general competencies of the teacher, digital competences of the student, student attitudes towards ICT, self-efficacy perceived and use of ICT resources. Interested universities can use this instrument to diagnose and improve the characteristics of both their human resources and technological infrastructure. The results obtained show the confidence of being a valid and reliable instrument for the measurement of the study variables. 
Miguel Angel Fitch Osuna

\title{
INTRODUCCIÓN
}

\begin{abstract}
lo largo de la historia de la humanidad la tecnología ha sido un factor trascendental, esto ha provocado una acelarada evolución que en decadas recientes nos ha llevado desde los conocimientos más fundamentales en electrónica y comunicaciones hasta lo que en un momento fue pensado inalcanzable y fantasioso. Sin embargo, no todas las economías del planeta siguen ese veloz ritmo, mientras que en unos países se habla de brecha digital en términos de fortalecer la infraestructura tecnológica, otros países discuten el comó aprovechar y obtener el mejor rendimiento de la tecnología para lograr sus objetivos a un menor esfuerzo en todos los ámbitos posibles.
\end{abstract}

En el caso particular del uso de las TIC en las instituciones de educación superior, lo cual puede ser altamente beneficioso para el desarrollo del capital humano, es impresindible investigar cuáles son los factores que favorecen el aprovechamiento de los recursos disponibles al alcance del estudiante y del profesor para un aprendizaje efectivo, estos pueden ser derivados de la falta de habilidades, conocimientos, actitudes y sentimientos que giran en torno al uso significativo y efectivo de los 
recursos digitales durante el ejercicio de la práctica educativa, así como los recursos implícitos en la operación y disponibilidad de la misma tecnología.

En la actualidad existen pocos estudios donde se tome una metodología para la construcción de un modelo con prueba de hipótesis cuantitativa, que demuestre la relación de cada una de las variables independientes tomadas en cuenta en esta investigación, en contraste con la variable dependiente situada bajo el contexto educativo. Ello en el caso particular de una institución pública de nivel superior, tomando en cuenta licenciaturas a fines al diseño de proyectos, como lo son la Licenciatura de Diseño Industrial y de Arquitectura, las cuales se ubicán en el estado de Nuevo León, México.

Para Claro, Sunkel y Trucco (2010), el impacto de las TIC en el aprendizaje guarda tres dimensiones, la prmera de ellas está en función de la relación entre el tipo de uso de la tecnología y los resultados de aprendizaje en asignaturas; la segunda dimensión se refiere a las condiciones pedagógicas y escolares que se usan las TIC y toma en cuenta el acceso adecuado a recursos TIC, integración por parte del profesor al curriculum y las condiciones institucionales a favor del uso de las TIC; por último el papel que juegan las características sociales e individuales del estudiante en su apropiación y forma de uso de las TIC, haciendo énfasis en que no sólo depende de las condiciones de disponibilidad que el estudiante tenga, sino su interacción con las TIC o dicho de otra manera, su capacidad de hacer uso de las oportunidades que estas ofrecen, relacionándose con factores como características cognitivas, culturales y sociodemográficas.

Para la Comisión Europea (2013) referente al impacto que tienen las TIC en el proceso de enseñanza y aprendizaje, son tomados en cuenta factores como el acceso, uso, actitudes, políticas y estrategias; esto para esclarecer el nivel de equipamiento, del comó y qué tan bien se usan, experiencias de aprendizajes de estudiantes y docentes, la confianza en su uso, así como las políticas y estrategias por parte de las escuelas; todo esto alrededor de su uso.

Por otra parte, resulta oportuno mencionar que el estudiante deberá de desarrollar una serie de actividades relacionadas con el uso, selección, utilización y organización de la información. Lo que le permitirá jugar un 
rol más participativo, produciendo y compartiendo conocimiento, algunas de las veces actuando como experto dentro de un marco colaborativo de aprendizaje, donde el soporte orientativo recibido por el docente será clave para el aprovechamiento de las TIC (Moreno y Delgado, 2013, p. 520).

Además, existen barreras que no le permiten a los docentes apreciar la incorporación de las TIC en las clases, como la falta de competencias tecnológicas por parte de los mismos para entender la funcionalidad de los programas básicos, el sistema operativo, además del uso efectivo del correo electrónico y navegadores de internet; poca disponibilidad de equipos o hardware y de conectividad al alcance de los interesados; ausencia de estrategias instruccionales tecnológicas que permitan la integración de contenidos digitales a los procesos de aprendizaje; falta de personal de apoyo técnico tanto a nivel de staff de computadoras y periféricos conectados, capaces de integrar las TIC al proceso de aprendizaje, como personal docente capacitado en la generación de contenidos digitales para el fortalecimiento del aprendizaje; la resistencia por parte del preofesor en el uso de las mismas por falta de confianza debido a que no existen recursos para su formación en métodos educativos que incorporen las TIC en la enseñanza (Araujo de Cendros y Bermudes, 2009, pp. 11-12).

García-Valcárcel y Tejedor (2010), señala que las dificultades de utilización de las TIC en la enseñanza es debido a la pobre capacitación de los docentes en el uso de la tecnología acorde a las necesidades del estudiante; carencia de tiempo del docente para la colaborar entre ellos y el desarrollo de programas de tecnología integrada; poca disponibilidad de personal de soporte tanto para problemas técnicos de cómputo, como de aplicación didáctica; insuficiente cantidad de ordenadores y acceso a internet en las aulas.

Derivado de lo anterior el interés de esta investigación, es contar como beneficio el empleo útil, provechoso o sacarle el máximo provecho, de todos aquellos recursos mediáticos concernientes a la tecnología informática, como las computadoras, los programas, recursos audiovisuales, acceso a internet, plataformas de apoyo, entre otros; utilizados por los estudiantes para fines formativos académicos, incluyendo el aprovechamiento del recurso humano que interfiere en el proceso de enseñanza, tomando en cuenta aquellas competencias que debe de reunir a favor del logro de los objetivos del perfil de egreso, siendo estos pertinentes al contexto tecnológico y el desempeño laboral; por su parte, Mesa (2012) 
aborda el concepto de pertinencia educativa y lo relaciona directamente con el aprendizaje al servicio del contexto y desarrollo local y señala que estos siempre están en función del desarrollo universal donde cada pueblo es una "Aldea Planetaria", de forma que los currículos pertinentes, y en general los procesos educativos, requieren también del uso apropiado de las Tecnologías de la Información y la Comunicación. De igual manera aporta que, de no existir una apropiación critica de las TIC, será un sistema desarticulado sin pertenencia con pocas oportunidades de aprendizaje, inequitativo y excluyente.

Tabla 1. Variables de investigación e indicadores de medición.

\begin{tabular}{|l|l|}
\hline Variable & Definición \\
\hline X1. Acceso de recursos & $\begin{array}{l}\text { Disponibilidad de medios tecnológicos y digitales } \\
\text { con que cuenta el alumno dentro y fuera del aula: } \\
\text { internet, equipo de cómputo adecuado, programas y } \\
\text { demás medios tecnológicos que sirven como apoyo al } \\
\text { proceso de enseñanza. (Claro, Sunkel, y Trucco, 2010, } \\
\text { p. 15); (Arteaga Sánchez y Duarte Hueros, 2010); } \\
\text { (Tejedor Tejedor y García-Valcárcel Muñoz-Repiso, } \\
\text { 2006). }\end{array}$ \\
\hline $\begin{array}{l}\text { X2. Actitud del estudian- } \\
\text { te ante las TIC }\end{array}$ & $\begin{array}{l}\text { Pensamientos, sentimientos y conductas que tiene } \\
\text { el estudiante hacia las TIC y que tienden a reflejarse } \\
\text { al momento de hablar, actuar y comportarse con los } \\
\text { demás. (Sarabia, 1994, p. 136); (Núñez Luna, Ochoa } \\
\text { Ávila, Vales García, Fernández Nista, y Ross Argüe- } \\
\text { lles, 2013); (Marcano B., Marcano, y Araujo, 2007, p. } \\
\text { 86). }\end{array}$ \\
\hline $\begin{array}{l}\text { X3. Competencias gene- } \\
\text { rales del docente }\end{array}$ & $\begin{array}{l}\text { Capacidad del docente para el desempeño satisfacto- } \\
\text { rio en su práctica pedagógica articulando sus múlti- } \\
\text { ples recursos personales (actitudes, conocimientos, } \\
\text { emociones, habilidades, valores...) y contextuales } \\
\text { (materiales, económicos, humanos...); (Gisbert } \\
\text { Cervera, Espuny Vidal, y González Martínez, 2011, p. } \\
\text { 76). } \\
\text { (Secretaría de Educación Pública, s.f.) (Hernandez } \\
\text { Suarez, Gamboa Suarez, y Ayala García, 2014, p. 6). }\end{array}$ \\
\hline
\end{tabular}




\begin{tabular}{|l|l|}
\hline X4. Competencias digita- & $\begin{array}{l}\text { Conjunto de conocimientos, habilidades, destrezas } \\
\text { y actitudes que debe lograr y desarrollar un docen- } \\
\text { te para hacer frente de manera óptima y critica la } \\
\text { incorporación, empleo y conocimiento de las TIC } \\
\text { dentro del proceso de enseñanza aprendizaje. (Rangel } \\
\text { Baca, 2015, p. 241); (Tourón, Martín, Navarro Asen- } \\
\text { sio, Pradas, y Iñigo, 2018, p. 28); (Ala-Mutka, Punie, } \\
\text { y Redecker, 2008, p. 1); (Hernández Suárez, Arévalo } \\
\text { Duarte, y Gamboa Suárez, 2016, p. 45). }\end{array}$ \\
\hline $\begin{array}{l}\text { X5. Competencias digita- } \\
\text { les del alumno }\end{array}$ & $\begin{array}{l}\text { Capacidad adquirida y desarrollada por el estudiante } \\
\text { para usar de manera eficaz, eficiente e innovadora } \\
\text { los instrumentos y recursos tecnológicos mediante } \\
\text { sus conocimientos, destrezas, actitudes y compor- } \\
\text { tamientos durante el proceso de enseñanza y apren- } \\
\text { dizaje. (Gisbert Cervera, Espuny Vidal, y González } \\
\text { Martínez, 2011, p. 76); (Arras Vota, Torres Gastelú, y } \\
\text { García-Valcárcel Muñoz-Repiso, 2011, pá 4). }\end{array}$ \\
\hline $\begin{array}{l}\text { X6. Autoeficacia perci- } \\
\text { bida }\end{array}$ & $\begin{array}{l}\text { Juicio que realiza a sí mismo el estudiante sobre sus } \\
\text { capacidades y habilidades para la ejecución y orga- } \\
\text { nización de acciones enfocadas a la realización de } \\
\text { actividades y proyectos académicos, de manera que le } \\
\text { permitan alcanzar el rendimiento deseado. (Peinado } \\
\text { de Briceño y Ramírez L., 2010, p. 23); (Véliz, Dorner, } \\
\text { y Sandoval, 2016, p. 99); (Blanco Vega, Martínez Ma- } \\
\text { rín, Zueck Enríquez, y Gastélum Cuadras, 2011, p. 3); } \\
\text { (Ornelas, Blanco, Rodríguez, y Flores, 2011, p. 23) }\end{array}$ \\
\hline Y. Aprovechamiento & $\begin{array}{l}\text { Grado de congruencia entre las expectativas del } \\
\text { estudiante en cuanto a su formación para el uso de las } \\
\text { TIC y su utilidad en el desempeño laboral. }\end{array}$ \\
\hline
\end{tabular}

Fuente: Elaboración propia.

En base a distintas investigaciones se adecuaron definiciones al contexto de nuestro interés y se definen las variables como se muestra en la Tabla 1.

Es por ello que esta investigación establece como hipótesis, que los factores relativos al acceso a recursos de TIC, la actitud del estudiante ante las TIC, las competencias generales del docente, las competencias digitales del docente, las competencias digitales del estudiante y la autoeficacia percibida del estudiante, están directamente relacionados con el aprovechamiento de las TIC orientados al perfil de egreso de los estudiantes 
de las licenciaturas de Arquitectura y Diseño Industrial; de esta manera se mantiene como objetivo el diseño de un instumento que permita la recolección de datos referenciados a las variables descritas anteriormente.

\section{MÉTODO}

La presente investigación está apegada al método científico, es del tipo exploratorio, debido a que desde el inicio de la investigación se exploró la literatura para fundamentar las variables involucradas con el aprovechamiento de los recursos tecnológicos; cuantitativo, descriptivo, ya que se realizó una recolecta de datos mediante indicadores en cada variable con la finalidad de analizar su incidencia e interrelación; con diseño no experimental, debido a que se observará el fenómeno en su estado natural; transversal, ya que se tomarán datos referentes a estudiantes de una misma generación que presentan el mismo programa de estudios, mismo examen de egreso y que coincidieron de alguna manera con la misma planta de docente.

\section{PROCEDiMiento}

Esta investigación se estructuró bajo los siguientes procedimientos:

1.Búsqueda en la literatura acerca de las variables relacionadas con el aprovechamiento de recursos TIC, conceptualizando y operacionalizando los constructos.

2.Se elaboró una recopilación de instrumentos de distintos investigadores, posteriormente adaptando y diseñando una primera versión.

3.Se sometió a revisión por expertos logrando a partir de las observaciones la validación del instrumento.

4.Se aplicó el instrumento en campo.

5.Se realizaron pruebas de confiabilización y validez de cosntructos.

\section{SE REALIZÓ ANÁLISIS DE LOS DATOS.}

Para el acopio de datos se utilizá un instrumento tipo encuesta Likert, considerando como unidad de análisis a los estudiantes próximos a egresar de la Facultad de Arquitectura de la UANL, en las licenciaturas de Diseño industrial y Arquitectura. La versión final consta de 70 preguntas divididas en 8 partes: datos generales ( 8 preguntas), para medir la actitud del estudiante ante las TIC (7 preguntas), para el acceso a recursos de 
TIC ( 8 preguntas), competencias generales del docente (14 preguntas), competencias digitales del docente ( 8 preguntas), competencias digitales del alumno ( 7 preguntas), autoeficacia percibida (14 preguntas), aprovechamiento (4 preguntas).

El origen de esta versión final tiene su punto de partida en la revisión de la literatura y la adaptación de otros instrumentos (Tabla 2), esto con la finalidad de no presentarse significados culturales diferentes y equivocadas interpretaciones, para lograr tal efecto, se sometió a validez de expertos donde se pretende eliminar aquellos items que son irreleva ntes y modifcar aquellos en que no esté claro su apego al concepto que se pretende medir y su relevancia con respecto a la investigación que se está llevando a cabo (Escobar Perez y Cuervo Martinez, 2008, pp. 27-28).

\begin{tabular}{|c|c|c|}
\hline Autores & Variable & $\begin{array}{l}\text { No. de } \\
\text { Îtems }\end{array}$ \\
\hline $\begin{array}{l}\text { (Acevedo Álvarez y Fer- } \\
\text { nández Díaz, 2004) }\end{array}$ & X3. Competencias generales del docente & $\begin{array}{l}26 \text { a } 29, \\
31 \text { a } 36\end{array}$ \\
\hline (Araiza Vázquez, 2009) & X1. Acceso a recursos TIC & $\begin{array}{l}16,17,21 \\
\text { a } 23\end{array}$ \\
\hline $\begin{array}{l}\text { (Jiménez González, Te- } \\
\text { rríquez Carrillo, y Robles } \\
\text { Zepeda, 2011) }\end{array}$ & X1. Acceso a recursos TIC & 19 \\
\hline $\begin{array}{l}\text { (Jiménez González, Te- } \\
\text { rríquez Carrillo, y Robles } \\
\text { Zepeda, 2011) }\end{array}$ & X3. Competencias generales del docente & $\begin{array}{l}24,25 \\
30,37\end{array}$ \\
\hline (Mirete Ruiz, 2016) & X5. Competencias digitales del alumno & $48,50,51$ \\
\hline (Mirete Ruiz, 2016) & X2. Actitud del estudiante ante las TIC & $\begin{array}{l}9 \text { a } 12, \\
14,15 \\
\end{array}$ \\
\hline $\begin{array}{l}\text { (Said Hung, Valencia } \\
\text { Cobos, y Silveira Sartori, } \\
\text { 2016) }\end{array}$ & X4. Competencias digitales del docente & $\begin{array}{l}38 \text { a } 41, \\
43,44\end{array}$ \\
\hline $\begin{array}{l}\text { (Said Hung, Valencia } \\
\text { Cobos, y Silveira Sartori, } \\
\text { 2016) }\end{array}$ & X5. Competencias digitales del alumno & 47 \\
\hline $\begin{array}{l}\text { (Universidad del Valle, } \\
\text { 2013) }\end{array}$ & $\begin{array}{l}\text { X1. Acceso a recursos TIC } \\
\text { Pertinencia al medio laboral }\end{array}$ & $\begin{array}{l}18,20 \\
67 \text { a } 70\end{array}$ \\
\hline
\end{tabular}




\begin{tabular}{|l|l|l|}
\hline $\begin{array}{l}\text { (Hernández Jácquez y } \\
\text { Barraza Macías, 2013) }\end{array}$ & X6. Autoeficacia percibida & $\begin{array}{l}53 \text { a 63, } \\
65,66\end{array}$ \\
\hline Elaboración propia & X2. Actitud del estudiante ante las TIC & 13 \\
\hline Elaboración propia & X4. Competencias digitales del docente & 42,45 \\
\hline Elaboración propia & X5. Competencias digitales del alumno & $46,49,52$ \\
\hline Elaboración propia & X6. Autoeficacia percibida & 64 \\
\hline
\end{tabular}

Fuente: Elaboración propia.

\section{ANÁLISIS CON EXPERTOS}

Una vez adaptados los items de otros cuestionarios, se revisó cuidadosamente la redacción de los mismos, ajustándolos al contexto de la investigación y tomando principal atención en su relevancia y representatividad al atributo que se deseaba medir. Para cuantificar la validez de contenido se realizó de acuerdo a Prat y Doval (2005), a través de índices de concordancia y relevancia sometido a evaluación por jueces los cuales debían reunir al menos dos de las siguientes características:

- Contar con un doctorado y practicar la docencia en las carreras de Arquitectura o Diseño Industrial.

- Investigar en áreas de conocimiento a fines de educación y aplicación de nuevas tecnologías.

- Laborar en el ámbito de la educación con la aplicación de las TIC.

Para fines concretos, 8 participantes reunieron el perfil requerido, dividiéndose en dos grupos, el primero constó de 4 participantes enfocados en la primera fase, dejando a los 4 restantes su participación para la segunda fase. A continuación se explica cómo se llevó a cabo cada una de ellas.

\section{FASE 1.-CLASIFICACIÓN Y UBICACIÓN DE LOS ÍTEMS EN UN CONSTRUCTO O VARIABLE.}

Primero se envió el instrumento junto con una serie de instrucciones a 4 jueces, los cuales recibieron la definición de cada uno de los constructos que deseábamos medir y el listado de ítems. El ejercicio consistía en relacionar cada ítem con cada constructo según la definición anexa. El resultado de esta primera fase consiste en eliminar aquellos ítems con un índice de concordancia menor a 3. Es decir, que al menos 3 de 4 investigadores relacionarán el ítem con su debida variable. 


\section{FASE 2.-EVAluación DE SU GRAdo de RELEVANCIA.}

Posteriormente se envió el instrumento al otro grupo de 4 jueces, con la finalidad de evaluar grado de relevancia de los ítems que superaron la primera etapa. La evaluación consistió en asignar un grado de importancia de dicho ítem para explicar un concepto. Es decir, cada uno se evaluó en una escala de uno a cuatro; donde uno es irrelevante, dos es poco relevante, tres es relevante y cuatro es muy relevante. Se realizó un cálculo de media para cada ítem, todos aquellos que obtuvieron un valor por debajo de tres fueron eliminados.

El resultado de estas actividades logró evitar polisemia o que los ítems tuvieran diferentes interpretaciones, así como evaluar la relevancia en cuanto las dimensiones de los constructos eliminando aquellos que no reunieran los requisitos de las dos pruebas antes descritas, obteniendo así el instrumento de investigación.

Para su aplicación, Hernández Sampieri y otros (2014, p. 174) señalan respecto a definfinir una población, esta debe establecerse claramente sus caracteristicas ayudando a delimitar los parametros muestrales.

De esta manera las consideraciones a tomar en cuenta para el presente estudio refieren a los alumnos que contarán con las siguientes características:

Ser alumnos vigentes de la Facultad de Arquitectura de la Universidad Autónoma de Nuevo León, ya sea en la licenciatura de Diseño Industrial o Arquitectura, que actualmente se encuentran realizando su último semestre.

Haber presentado el examen general de egreso ya sea EGEL o EXENS en el semestre agosto diciembre 2017.

Se tomaron en cuenta como población, 313 alumnos evaluados en el semestre agosto diciembre 2017, inscritos en las licenciatura de Arquitectura y Diseño Industrial, de lo cual se obtuvo una muestra de 173 estudiantes, esto por muestreo probabilístico, ya que cualquier estudiante que cumpla con las especificaciones antes descritas, guardaba la misma posibilidad para aplicarle el instrumento de recolección de datos. 


\section{RESULTADOS}

\section{COFIABILIDAD}

Posterior a esto, se procedió a evaluar la confiablidad del instrumento, consistente en saber a qué grado el instrumento de medición aplicado en repetidas ocasiones produce los mismos resultados (Mendoza y Garza, 2009, p. 30). A través del programa Smartpls se realizó el análisis de confiabilidad de la consistencia interna mediante Alpha de Cronbach, la cual varía de 0 a 1 . Valores de confiabilidad de 0.60 a 0.90 pueden ser considerados como satisfactorios para investigaciones exploratorias, mientras que para investigaciones más avanzadas valores entre $0.70 \mathrm{y}$ 0.90 son satisfactorios. Valores por encima de 0.90 (sobre todo por encima de 0.95 ) no son deseables ya que muestran que todos los indicadores de la variable están midiendo el mismo fenómeno y por lo tanto es muy probable que no sean una medida válida de construcción (Hair, Hult, Ringle, y Sarstedt, 2014, p. 102).

Mediante las siguientes tablas podemos observar que los constructos de las variables son confiables en su mayoría debido a que se encuentran dentro de un rango satisfactorio, tanto en los valores de Alfa de Cronbach y de fiabilidad compuesta (Tabla 3). Sólo en algunos casos se mostraron valores por encima de 0.9 sin llegar a sobrepasar 0.95 y los demás se encontraron en niveles satisfactorios.

Tabla 3. Consistencia interna del instrumento (Alpha de Cronbach y fiabilidad compuesta)

\begin{tabular}{|l|l|l|l|l|}
\hline Variables & $\begin{array}{l}\text { No. de pre- } \\
\text { guntas origi- } \\
\text { nales }\end{array}$ & $\begin{array}{l}\text { Alfa de Cron- } \\
\text { bach }\end{array}$ & $\begin{array}{l}\text { Fiabilidad } \\
\text { compuesta }\end{array}$ & $\begin{array}{l}\text { No. de pre- } \\
\text { guntas que } \\
\text { se elimina- } \\
\text { ron }\end{array}$ \\
\hline $\begin{array}{l}\text { X1 - Acceso a recur- } \\
\text { sos de TIC }\end{array}$ & 7 & 0.774 & 0.835 & 0 \\
\hline $\begin{array}{l}\text { X2 - Actitud del estu- } \\
\text { diante ante las TIC }\end{array}$ & 8 & 0.771 & 0.825 & 0 \\
\hline $\begin{array}{l}\text { X3 - Competencias } \\
\text { Generales Docente }\end{array}$ & 14 & 0.936 & 0.944 & 0 \\
\hline
\end{tabular}




\begin{tabular}{|l|l|l|l|l|}
\hline $\begin{array}{l}\text { X4 - Competencias } \\
\text { Digitales Docente }\end{array}$ & 8 & 0.907 & 0.925 & 0 \\
\hline $\begin{array}{l}\text { X5 - Competencias } \\
\text { Digitales Alumno }\end{array}$ & 7 & 0.798 & 0.841 & 0 \\
\hline $\begin{array}{l}\text { X6 - Autoeficacia } \\
\text { Percibida }\end{array}$ & 14 & 0.9 & 0.913 & 0 \\
\hline Y - Aprovechamiento & 4 & 0.873 & 0.913 & 0 \\
\hline
\end{tabular}

Fuente: Elaboración propia mediante utilización de programa SmartPLS

La confiabilidad del indicador, se realiza mediante las cargas externas del constructo y significa cuánto de la varianza de un ítem se explica por el constructo y éste debe ser altamente significativo o al menos con un valor mínimo del $50 \%$ explicativo. Esto resulta debido a que las cargas externas son el cuadrado de la varianza explicada por el constructo, de tal manera, que si se desea explicar al menos el 50\%, la carga debe de ser un valor como mínimo de 0.708 , ya que este número (0.708) es la raíz cuadrada de 0.50 . Aquellos indicadores que estén entre valores de 0.70 y 0.40 , se deberán considerar eliminarlos si esto produce una alza en la fiabilidad compuesta o si afecta la validez de contenido. En cuanto a los indicadores por debajo de 0.40, deberán ser eliminados (Hair, Hult, Ringle, y Sarstedt, 2014, p. 103).

En la Tabla 4, se muestra la confiablidad del indicador y se identifica cuáles están entre un rango de 0.70 y 0.40 para considerar su eliminación.

Tabla 4. Cargas externas de los constructos y sus indicadores

\begin{tabular}{|c|c|l|l|l|l|l|l|}
\hline & $\begin{array}{l}\text { Acceso de } \\
\text { recursos de } \\
\text { TIC }\end{array}$ & $\begin{array}{l}\text { Actitudes del } \\
\text { estudiante } \\
\text { ante las TIC }\end{array}$ & $\begin{array}{l}\text { Aprove- } \\
\text { chamiento } \\
\text { de los } \\
\text { recursos } \\
\text { TIC }\end{array}$ & $\begin{array}{l}\text { Autoe- } \\
\text { ficacia } \\
\text { percibida }\end{array}$ & $\begin{array}{l}\text { Competen- } \\
\text { cias digitales } \\
\text { del alumno }\end{array}$ & $\begin{array}{l}\text { Competen- } \\
\text { cias digitales } \\
\text { del docente }\end{array}$ & $\begin{array}{l}\text { Competencias } \\
\text { generales del } \\
\text { docente }\end{array}$ \\
\hline ACC1 & 0.77 & & & & & & \\
\hline ACC2 & 0.799 & & & & & & \\
\hline ACC3 & 0.774 & & & & & & \\
\hline ACC4 & 0.797 & & & & & & \\
\hline ACC5 & 0.564 & & & & & & \\
\hline ACC8 & 0.596 & & & & & & \\
\hline ACT1 & & 0.834 & & & & & \\
\hline ACT2 & & 0.881 & & & & & \\
\hline ACT3 & & 0.772 & & & & & \\
\hline ACT7 & & 0.793 & & & & & \\
\hline AEP1 & & & & 0.661 & & & \\
\hline
\end{tabular}




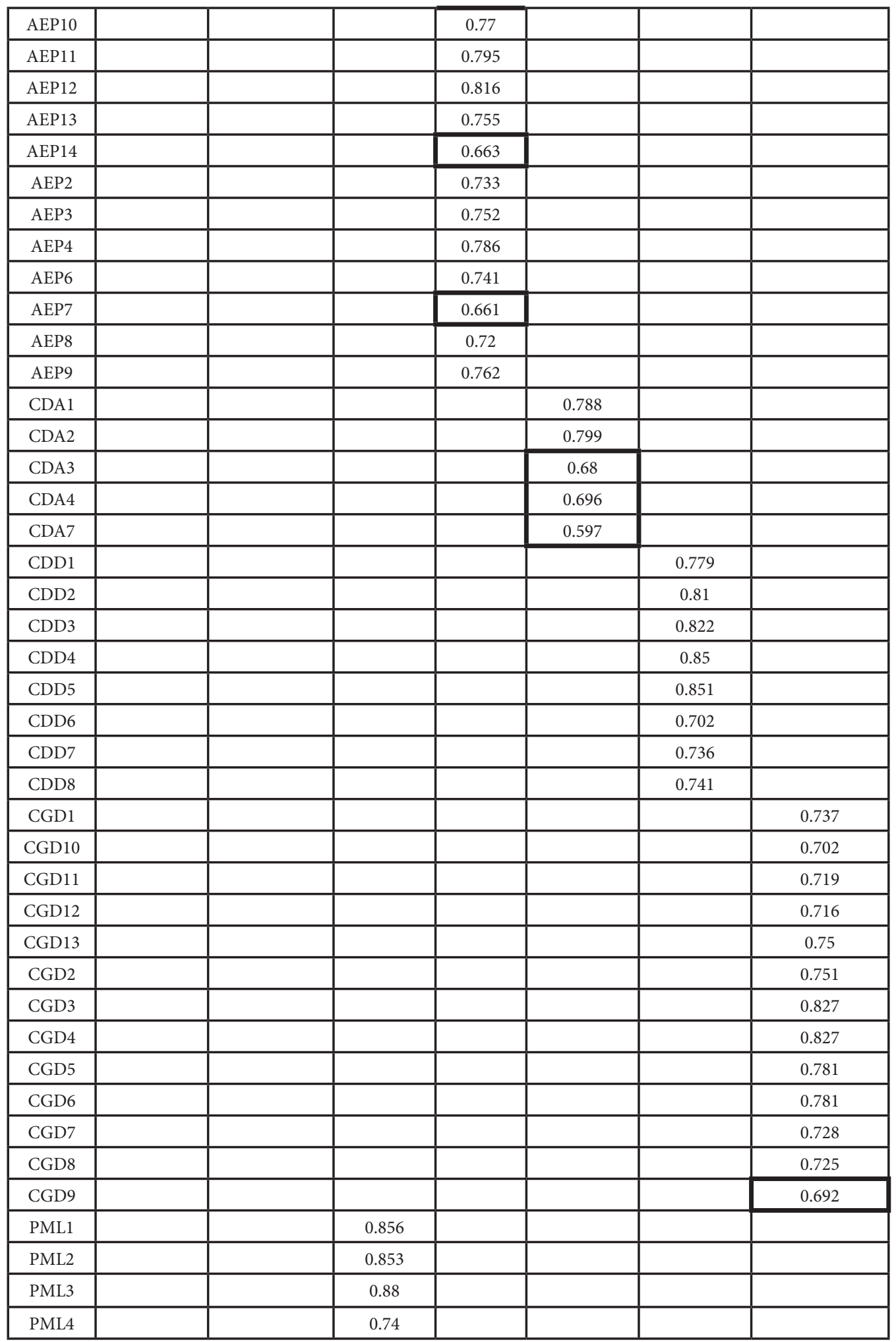

Fuente: Elaboración propia mediante resultados de SmartPLS 
La validez convergente es el grado en que una medida se correlaciona positivamente con medidas alternativas del mismo constructo. En otras palabras, evalúa el grado en que la medida de los ítems que recogen un mismo concepto y están correlacionados. Si esta correlación es alta, entonces se está midiendo el concepto deseado. Para tal efecto se puede utilizar la varianza media extraída (AVE). Este criterio se define comó el gran valor medio de las cargas al cuadrado de los indicadores asociados con el constructo, explicado de otra manera, la suma del cuadrado de las cargas entre el número de indicadores. De manera similar a los indicadores individuales, se busca que se explique al menos la mitad de la varianza, de tal manera que valores AVE iguales o mayores que 0.50 resultan aceptables (Hair et al., 2014, p.103; Kwong, 2013, p.22).

A continuación en la Tabla 5, se encuentra que todos los valores AVE son mayores que el umbral aceptable de 0.5 , por lo que se confirma la validez convergente.

Tabla 5. Varianza extraída media AVE

\begin{tabular}{|l|l|}
\hline Constructo & AVE \\
\hline X1 - Acceso a recursos de TIC & 0.523 \\
\hline X2 - Actitudes del estudiante ante las TIC & 0.674 \\
\hline X3 - Competencias Generales del Docente & 0.563 \\
\hline X4 - Competencias Digitales del Docente & 0.621 \\
\hline X5 - Competencias Digitales del Alumno & 0.513 \\
\hline X6 - Autoeficacia Percibida & 0.549 \\
\hline Y - Aprovechamiento & 0.696 \\
\hline
\end{tabular}

Fuente: Elaboración propia mediante resultados de SmartPLS

El método para evaluar la validez discriminante mediante el examen de cargas cruzadas de los indicadores consiste en comparar la carga externa de un indicador en el constructo asociado, siendo esta mayor a la carga en otros constructos (es decir, las cargas transversales). La presencia de cargas cruzadas que exceden las cargas externas de los indicadores representa un problema de validez discriminante (Hair, Hult, Ringle, y Sarstedt, 2014, p. 105).

Tabla 6. Cargas cruzadas (cross loadings)

\begin{tabular}{|l|l|l|l|l|l|l|l|}
\hline $\begin{array}{l}\text { Indica- } \\
\text { dor }\end{array}$ & $\begin{array}{l}\text { Acceso a recur- } \\
\text { sos de TIC }\end{array}$ & $\begin{array}{l}\text { Actitudes del } \\
\text { estudiante } \\
\text { ante las TIC }\end{array}$ & $\begin{array}{l}\text { Aprovecha- } \\
\text { miento }\end{array}$ & $\begin{array}{l}\text { Autoe- } \\
\text { ficacia } \\
\text { Percibida }\end{array}$ & $\begin{array}{l}\text { Compe- } \\
\text { tencias } \\
\text { Digitales del } \\
\text { Alumno }\end{array}$ & $\begin{array}{l}\text { Competen- } \\
\text { cias Digitales } \\
\text { del Docente }\end{array}$ & $\begin{array}{l}\text { Competencias } \\
\text { Generales del } \\
\text { Docente }\end{array}$ \\
\hline ACC1 & 0.77 & 0.156 & 0.337 & 0.264 & 0.172 & 0.409 & 0.407 \\
\hline
\end{tabular}




\begin{tabular}{|c|c|c|c|c|c|c|c|}
\hline ACC2 & 0.799 & 0.219 & 0.294 & 0.29 & 0.179 & 0.421 & 0.446 \\
\hline ACC 3 & 0.774 & 0.189 & 0.287 & 0.232 & 0.106 & 0.356 & 0.439 \\
\hline ACC4 & 0.797 & 0.252 & 0.315 & 0.324 & 0.236 & 0.397 & 0.487 \\
\hline ACC5 & 0.564 & 0.115 & 0.324 & 0.232 & 0.146 & 0.346 & 0.308 \\
\hline ACC8 & 0.596 & 0.237 & 0.297 & 0.315 & 0.306 & 0.394 & 0.367 \\
\hline ACT1 & 0.245 & 0.834 & 0.049 & 0.196 & 0.066 & 0.147 & 0.305 \\
\hline ACT2 & 0.204 & 0.881 & 0.173 & 0.204 & 0.144 & 0.21 & 0.307 \\
\hline АCT3 & 0.24 & 0.772 & 0.164 & 0.246 & 0.019 & 0.242 & 0.311 \\
\hline ACT7 & 0.212 & 0.793 & 0.191 & 0.324 & 0.095 & 0.145 & 0.302 \\
\hline AEP1 & 0.315 & 0.227 & 0.285 & 0.661 & 0.403 & 0.305 & 0.304 \\
\hline AEP10 & 0.243 & 0.207 & 0.281 & 0.77 & 0.336 & 0.362 & 0.382 \\
\hline AEP11 & 0.32 & 0.262 & 0.329 & 0.795 & 0.313 & 0.472 & 0.468 \\
\hline AEP12 & 0.349 & 0.264 & 0.368 & 0.816 & 0.467 & 0.439 & 0.382 \\
\hline AEP13 & 0.275 & 0.252 & 0.284 & 0.755 & 0.284 & 0.325 & 0.338 \\
\hline AEP14 & 0.275 & 0.235 & 0.31 & 0.663 & 0.212 & 0.326 & 0.34 \\
\hline AEP2 & 0.333 & 0.157 & 0.281 & 0.733 & 0.378 & 0.305 & 0.352 \\
\hline AEP3 & 0.3 & 0.189 & 0.342 & 0.752 & 0.345 & 0.251 & 0.283 \\
\hline AEP4 & 0.333 & 0.253 & 0.275 & 0.786 & 0.424 & 0.332 & 0.315 \\
\hline AEP6 & 0.217 & 0.146 & 0.29 & 0.741 & 0.306 & 0.314 & 0.321 \\
\hline AEP7 & 0.15 & 0.142 & 0.244 & 0.661 & 0.253 & 0.378 & 0.352 \\
\hline AEP8 & 0.252 & 0.215 & 0.351 & 0.72 & 0.327 & 0.411 & 0.451 \\
\hline AEP9 & 0.282 & 0.292 & 0.264 & 0.762 & 0.331 & 0.431 & 0.385 \\
\hline CDA1 & 0.262 & 0.086 & 0.364 & 0.35 & 0.788 & 0.353 & 0.332 \\
\hline CDA2 & 0.128 & 0.002 & 0.288 & 0.371 & 0.799 & 0.257 & 0.231 \\
\hline CDA3 & 0.175 & 0.165 & 0.28 & 0.358 & 0.68 & 0.262 & 0.205 \\
\hline CDA4 & 0.203 & 0.031 & 0.377 & 0.299 & 0.696 & 0.343 & 0.297 \\
\hline CDA7 & 0.185 & 0.083 & 0.185 & 0.305 & 0.597 & 0.169 & 0.184 \\
\hline CDD1 & 0.445 & 0.23 & 0.485 & 0.362 & 0.264 & 0.779 & 0.636 \\
\hline CDD2 & 0.417 & 0.154 & 0.438 & 0.274 & 0.349 & 0.81 & 0.567 \\
\hline CDD3 & 0.471 & 0.191 & 0.539 & 0.374 & 0.36 & 0.822 & 0.523 \\
\hline CDD4 & 0.486 & 0.205 & 0.507 & 0.455 & 0.327 & 0.85 & 0.662 \\
\hline CDD5 & 0.409 & 0.227 & 0.467 & 0.469 & 0.338 & 0.851 & 0.545 \\
\hline CDD6 & 0.286 & 0.152 & 0.405 & 0.377 & 0.276 & 0.702 & 0.512 \\
\hline CDD7 & 0.367 & 0.077 & 0.468 & 0.283 & 0.236 & 0.736 & 0.52 \\
\hline CDD8 & 0.471 & 0.196 & 0.48 & 0.425 & 0.332 & 0.741 & 0.546 \\
\hline CGD1 & 0.504 & 0.349 & 0.498 & 0.398 & 0.379 & 0.519 & 0.737 \\
\hline CGD10 & 0.418 & 0.258 & 0.379 & 0.392 & 0.236 & 0.551 & 0.702 \\
\hline CGD11 & 0.396 & 0.168 & 0.489 & 0.278 & 0.195 & 0.526 & 0.719 \\
\hline CGD12 & 0.282 & 0.204 & 0.358 & 0.261 & 0.239 & 0.463 & 0.716 \\
\hline CGD13 & 0.342 & 0.189 & 0.42 & 0.254 & 0.234 & 0.589 & 0.75 \\
\hline CGD2 & 0.519 & 0.403 & 0.463 & 0.394 & 0.336 & 0.576 & 0.751 \\
\hline CGD3 & 0.496 & 0.344 & 0.5 & 0.386 & 0.229 & 0.559 & 0.827 \\
\hline CGD4 & 0.428 & 0.355 & 0.385 & 0.379 & 0.282 & 0.559 & 0.827 \\
\hline CGD5 & 0.446 & 0.297 & 0.351 & 0.456 & 0.228 & 0.531 & 0.781 \\
\hline CGD6 & 0.523 & 0.383 & 0.509 & 0.44 & 0.256 & 0.529 & 0.781 \\
\hline CGD7 & 0.446 & 0.214 & 0.381 & 0.4 & 0.246 & 0.493 & 0.728 \\
\hline
\end{tabular}




\begin{tabular}{|l|l|l|l|l|l|l|l|}
\hline CGD8 & 0.318 & 0.187 & 0.424 & 0.309 & 0.296 & 0.571 & 0.725 \\
\hline CGD9 & 0.327 & 0.152 & 0.352 & 0.268 & 0.288 & 0.541 & 0.692 \\
\hline PML1 & 0.376 & 0.131 & 0.856 & 0.389 & 0.436 & 0.466 & 0.453 \\
\hline PML2 & 0.379 & 0.155 & 0.853 & 0.37 & 0.361 & 0.576 & 0.51 \\
\hline PML3 & 0.357 & 0.145 & 0.88 & 0.314 & 0.408 & 0.548 & 0.513 \\
\hline PML4 & 0.314 & 0.185 & 0.74 & 0.276 & 0.182 & 0.408 & 0.432 \\
\hline
\end{tabular}

Fuente: Elaboración propia mediante resultados de SmartPLS

Al observar la Tabla 6, podemos corroborar que las cargas cruzadas tienen la mayor carga en su respectivo constructo.

El crtiterio de Fornell y Larcker consiste en que la raíz cuadrada de AVE en cada variable latente se puede utilizar para establecer la validez discriminante, si este valor es mayor que otros valores de correlación entre las variables latentes. La lógica de este método se basa en la idea de que un constructo comparte más varianza con sus indicadores asociados que con cualquier otro constructo (Hair et al., 2014, p. 105; Kwong, 2013, p. 22).

Tabla 7. Coorelaciones de Fornell-Larcker

\begin{tabular}{|l|l|l|l|l|l|l|l|}
\hline & $\begin{array}{l}\text { Acceso de } \\
\text { recursos } \\
\text { de TIC }\end{array}$ & $\begin{array}{l}\text { Actitudes } \\
\text { del es- } \\
\text { tudiante } \\
\text { ante las } \\
\text { TIC }\end{array}$ & $\begin{array}{l}\text { Aprovecha- } \\
\text { miento de } \\
\text { los recursos } \\
\text { TIC }\end{array}$ & $\begin{array}{l}\text { Autoe- } \\
\text { ficacia } \\
\text { percibida }\end{array}$ & $\begin{array}{l}\text { Compe- } \\
\text { tencias di- } \\
\text { gitales del } \\
\text { alumno }\end{array}$ & $\begin{array}{l}\text { Compe- } \\
\text { tencias } \\
\text { digitales } \\
\text { del do- } \\
\text { cente }\end{array}$ & $\begin{array}{l}\text { Compe- } \\
\text { tencias ge- } \\
\text { nerales del } \\
\text { docente }\end{array}$ \\
\hline $\begin{array}{l}\text { Acceso de } \\
\text { recursos de } \\
\text { TIC }\end{array}$ & 0.723 & & & & & & \\
\hline $\begin{array}{l}\text { Actitudes } \\
\text { del estu- } \\
\text { diante ante } \\
\text { las TIC }\end{array}$ & 0.274 & 0.821 & & & & & \\
\hline $\begin{array}{l}\text { Aprovecha- } \\
\text { miento de } \\
\text { los recursos } \\
\text { TIC }\end{array}$ & 0.428 & 0.181 & 0.834 & & & & \\
\hline $\begin{array}{l}\text { Autoe- } \\
\text { ficacia } \\
\text { percibida }\end{array}$ & 0.386 & 0.296 & 0.406 & 0.741 & & & \\
\hline $\begin{array}{l}\text { Compe- } \\
\text { tencias } \\
\text { digitales } \\
\text { del alumno }\end{array}$ & 0.268 & 0.1 & 0.426 & 0.47 & 0.716 & & \\
\hline
\end{tabular}




\begin{tabular}{|l|l|l|l|l|l|l|l|}
\hline $\begin{array}{l}\text { Compe- } \\
\text { tencias } \\
\text { digitales } \\
\text { del docente }\end{array}$ & 0.539 & 0.23 & 0.604 & 0.481 & 0.396 & 0.788 & \\
\hline $\begin{array}{l}\text { Compe- } \\
\text { tencias } \\
\text { generales } \\
\text { del docente }\end{array}$ & 0.572 & 0.373 & 0.573 & 0.481 & 0.355 & 0.718 & 0.75 \\
\hline
\end{tabular}

Nota: La raíz cuadrada del AVE supera las correlaciones existentes con los demás constructos. Fuente: Elaboración propia mediante resultados de SmartPLS

Bajo este criterio, podemos apreciar en la Tabla 7, cómo el valor es mayor que los valores de correlación entre los otros constructos, estableciendo la existencia de validez discriminante entre variables.

\section{DISCUSIÓN}

El actual dinamismo económico en la sociedad del conocimiento y la información, exige a las instituciones de nivel superior focalizar sus esfuerzos hacia el aprovechamiento tanto de las infraestructuras técnologicas como del recurso humano, donde el acceso, actitudes, habilidades y competencias tanto de alumnos como de los docentes juegan un papel fundamental. En este sentido, fue necesario conceptualizar estos factores necesarios en el estudiante, cumpliendo con lo señalado en su perfil de egreso. Por otro lado, resulta cuestionable, cuáles son las competencias qué debe de contar el docente para lograr resultados significativos en la educación de sus estudiantes. El propósito de este estudio es formular indicadores que nos permitan la construcción de un instrumento que nos lleve a medir a qué grado los factores considerados intervienen con el aprovechamiento de los recursos tecnologicos en las instituciones de nivel superior.

Es por ello, que debe existir una constante evaluación de las competencias docentes, tanto en lo digital como en lo pedagógico, ya que juegan un papel fundamental en la creación de ambientes de aprendizaje propiciando con sus actitudes y aptitudes el logro de resultados académicos provechosos. El contar con instrumentos de este tipo contribuyen a evaluar las áreas de oportunidad que se presentan en el contexto educativo, facilitando el redireccionamiento de recursos hacia los programas de estudio, planes de formación docente, infraestructura tecnológica, fortalecer alguna herramienta en particular o abordar alguna solución puntual hacia la formación de egresados competentes. 
En cuanto a los resultados obtenidos, se demostró que el modelo propuesto cumple en cuanto a los valores aceptables de fiabilidad, consistencia interna y validez, en consecuencia, nos ayuda a evidenciar de manera parcial la visión que guardan los estudiantes hacía los distintos factores que comprenden su educación profesional. Por otra parte, es necesario aclarar que los resultados obtenidos obedecen a una muestra y un contexto determinado, sugiriendo su investigación adicional para muestras y contextos diferentes.

Este instrumento será de gran utilidad para aquellas instituciones de nivel superior que quieran diagnosticar su situación en base a los factores analizados en este estudio, enfocados en la innovación tecnologica dentro de un marco pedagógico, de igual manera, de acuerdo a las garantías de fiabilidad y validez, este instrumento ofrece un punto de partida y herramienta útil para profundizar en el tema. Será necesario, en futuras investigaciones explorar otras tecnologías, tomar en cuenta la opinión del estudiante en cuanto a sus expectativas en los entornos digitales y su pertinencia al medio laboral.

\section{REFERENCIAS}

Ala-Mutka, K., Punie, Y. y Redecker, C. (2008). Digital Competence for. JRC Technical notes, 1-5. Obtenido de http://ftp.jrc.es/EURdoc/JRC48708.TN.pdf

Araujo de Cendros, D. y Bermudes, J. (2009). Limitaciones de las tecnologías de información y comunicación en la educación universitaria. Horizontes Educacionales, 14(1), 9-24.

Arras Vota, A., Torres Gastelú, C. y García-Valcárcel Muñoz-Repiso, A. (2011). Competencias en Tecnologías de Información y Comunicación (TIC) de los estudiantes universitarios. Revista Latina de Comunicación Social, 130 - 111.

Blanco Vega, H., Martínez Marín, M., Zueck Enríquez, M. y Gastélum Cuadras, G. (2011). ANÁLISIS PSICOMÉTRICO DE LA ESCALA AUTOEFICACIA EN CONDUCTAS ACADÉMICAS EN UNIVERSITARIOS DE PRIMER INGRESO. Revista Electrónica Actualidades, 11(3), 1-27.

Centeno Moreno, G. y Cubo Delgado, S. (2013). Evaluación de la competencia digital y las actitudes hacia las TIC del alumnado universitario. Revista de Investigación Educativa, 517-536. 
Claro, M., Sunkel, G. y Trucco, D. (2010). Impacto de las TIC en los aprendizajes de los estudiantes. Estado del arte. CEPAL - Colección docmentos de proyectos, 1-28.

Escobar Perez, J. y Cuervo Martinez, A. (2008). Validez de contenido y juicio de expertos: Una aproximación a su utilización. Avances de medición, (6), 27-36.

European Commission. (2013). Survey of schools: ICT in education, benchmarking access, use and attitudes to technology in Europe's schools. Belgium: Publications Office of the European Union.

García-Valcárcel Muñoz-Repiso, A. y Tejedor Tejedor, F. (2010). Evaluación de procesos de innovación escolar basados en el uso de las TIC desarrollados en la comunidad de Castilla y León. Revista de educación, 125-147.

Gisbert Cervera, M., Espuny Vidal, C. y González Martínez, J. (2011). Incotic. Una herramienta para la autoevaluación diagnostica de la competencia digital en la Universidad. Profesorado, Revista de curriculum y formación del profesorado., 74-90.

Hair, J., Hult, G., Ringle, C. y Sarstedt, M. (2014). A primer on Partial Least Squares Structural Equation Modeling (PLS-SEM). Thousand Oaks, Los Angeles: SAGE Publications, Inc.

Hernández Sampieri, R., Fernandez Collado, C. y Baptista Lucio, M. (2014). Métodología de la investigación. México D.F.: Mc Graw Hill.

Hernández Suárez, C., Arévalo Duarte, M. y Gamboa Suárez, A. (2016). Competencias TIC para el desarrollo profesional docente en educación básica. Praxis \& Saber, 7(14), 41-69.

Hernandez Suarez, C., Gamboa Suarez, A. y Ayala García, E. (Noviembre de 2014). Competencias para los docentes de educación superior. (OEI, Ed.) Congreso Iberoamericano de Ciencia, Tecnología, Innovación y Educación. Obtenido de http://www.oei.es/ congreso2014/memoriactei/837.pdf

Ken Kwong, K. (2013). Partial Least Squares Structural Equation Modeling (PLS-SEM) Techniques Using SmartPLS. Marketing Bulletin, 1-32.

Marcano B., Marcano, N. y Araujo, D. (2007). Actitud de los estudiantes de los institutos universitarios frente a las tecnologías de la información y la comunicación. Telématique, 6(1), 77-106.

Mendoza, J. y Garza, J. (2009). La medición en el proceso de investigación científica: Evaluación de validez de contenido y confiabilidad. Innovaciones de Negocios, 6(1), 17-32. 
Mesa Agudelo, W. (2012). Las TIC como herramientas potenciadoras de equidad, pertinencia e inclusión educativa. Revista Trilogía, 61-77.

Núñez Luna, D., Ochoa Ávila, E., Vales García, J., Fernández Nista, M. y Ross Argüelles, G. (2013). Actitudes y hábitos asociados al uso de las TICs en alumnos de psicología. Psicología para América Latina, 91-114.

Ornelas, M., Blanco, H., Rodríguez, J. y Flores, F. (2011). Análisis Psicométrico de la Escala Autoeficacia en Conductas de Cuidado de la Salud Física en Universitarios de Primer Ingreso. Formación Universitaria, 4(6), 21-34. doi:10.4067/S0718-50062011000600004

Peinado de Briceño, S. y Ramírez L. (2010). Adaptación de un instrumentopara evaluar la autoeficacia computacional en estudiantes venezolanos. Revista Enseñanza e Investigación en Psicología, 15(1), 21-30.

Prat, S. y Doval, D. (2005). Construcción y análisis de escalas. Análisis Multivariable para las Ciencias Sociales. Madrid: Pearson Prentice Hall.

Rangel Baca, A. (2015). Competencias docentes digitales: propuesta de un perfil. Píxel-Bit. Revista de Medios y Educación(46), 235 a 248. doi:http://dx.doi.org/10.12795/ pixelbit.2015.i46.15

Sarabia, B. (1994). El aprendizaje y la enseñanza de las actitudes. En C. Coll, J. I. Pozo, B. Sarabia, \& E. Valls, Los contenidos de la reforma. Enseñanza y aprendizaje de conceptos, procedimientos y actitudes (págs. 133-198). Mexico D.F.: Santillana

Tejedor Tejedor, F. y García-Valcárcel Muñoz-Repiso, A. (2006). Competencia de los profesores para el uso de las TIC en la enseñanza. Análisis de sus conocimientos y actitudes. Revista española de pedagogía, 21-44.

Tourón, J., Martín, D., Navarro Asensio, E., Pradas, S., \& Iñigo, V. (2018). Validación de constructo de un instrumento para medir la competencia digital docente de los profesores(CDD). Revista Española de Pedagogía, 76(269), 25-54. doi:10.22550/ REP76-1-2018-02 